\title{
Fuzzy Group Decision Making for Influence-Aware Recommendations
}

\author{
Nicola Capuano \\ Dep. of Information, Electric Engineering and Applied Mathematics, University of Salerno \\ Via Giovanni Paolo II 132, 84084 Fisciano (SA), Italy. (e-mail: ncapuano@unisa.it) \\ Francisco Chiclana \\ School of Computer Science and Informatics, Faculty of Technology, De Montfort University \\ Leicester LE1 9BH, UK (e-mail: chiclana@dmu.ac.uk) \\ Enrique Herrera-Viedma \\ Dep. of Computer Science and Artificial Intelligence, University of Granada \\ Granada 18071, Spain (e-mail: viedma@decsai.ugr.es)

\section{Hamido Fujita} \\ Faculty of Software and Information Science, Iwate Prefectural University \\ Iwate, Japan (e-mail: HFujita-799@acm.org) \\ Vincenzo Loia ${ }^{1}$ \\ Dep. of Management and Innovation Systems, University of Salerno \\ Via Giovanni Paolo II 132, 84084 Fisciano (SA), Italy (e-mail: Ioia@unisa.it)
}

${ }^{1}$ Corresponding Author 


\title{
Fuzzy Group Decision Making for Influence-Aware Recommendations
}

\begin{abstract}
Group Recommender Systems are special kinds of Recommender Systems aimed at suggesting items to groups rather than individuals taking into account, at the same time, the preferences of all (or the majority of) members. Most existing models build recommendations for a group by aggregating the preferences for their members without taking into account social aspects like user personality and interpersonal trust, which are capable of affecting the item selection process during interactions. To consider such important factors, we propose in this paper a novel approach to group recommendations based on fuzzy influence-aware models for Group Decision Making. The proposed model calculates the influence strength between group members from the available information on their interpersonal trust and personality traits (possibly estimated from social networks). The estimated influence network is then used to complete and evolve the preferences of group members, initially calculated with standard recommendation algorithms, toward a shared set of group recommendations, simulating in this way the effects of influence on opinion change during social interactions. The proposed model has been experimented and compared with related works.
\end{abstract}

Keywords: recommender systems, group decision making, social influence

\section{Introduction}

In recent years, Recommender Systems (RSs) have become increasingly popular to support several human activities by anticipating needs and generating personalized suggestions for users. They are currently adopted in a variety of areas for the selection of items like movies, music, news, books, courses, papers, products, etc. [1, 2, 3, 4]. Even if the majority of RSs provides recommendations for individual users, there are activities that can be performed by groups of people, like watching a movie, going to a restaurant or traveling with friends. In such cases, recommendations should be aimed at groups rather than individuals and the preferences of all (or the majority of) group members should be taken into account [5].

Therefore, Group Recommender Systems (GRSs) are RSs addressed to groups of users. In addition to the scenarios mentioned above, they can play an important role in Ambient Intelligence, supporting applications that sense the environment and respond to the presence of people with personalized content [6]. As most physical environments are shared by many people at the same time, once their profiles are inferred or retrieved (e.g. via sensors, smart devices, RFID systems, etc.) GRSs can be employed to select the most feasible content that fits all preferences. Examples include the selection of the products to advertise on digital signage or the background music to be played in physical stores to maximize customers well-being and, ultimately, increasing sales.

The majority of existing GRS approaches are based on the aggregation of recommendations generated for individual group members by a standard RS. Nevertheless, in many contexts, social factors such as user personality, influence and mutual relationships play an important role in the final decision adopted by a group [7]. In order to take these factors into account, we propose a new model for group recommendations based on Group Decision Making (GDM) $[8,9]$. The proposed model measures interpersonal trust and users' personality traits (possibly by analyzing data gathered from popular social networks like Facebook or Twitter) and use them to estimate the influence strength between group members. Then, by relying on such information, the individual preferences of group members (obtained through a standard RS) are completed (in case of limited coverage of RS data) and evolved toward a shared decision simulating the effects of social influence on opinion change [10,11].

The simulation of opinions evolution thanks to social interaction is a distinctive feature of the proposed model as well as the adoption of a GDM-based approach. Moreover, differently from other 
existing GRSs, the proposed model is able to take into account the effects of both interpersonal trust and individual personality traits that are evaluated transparently, without the need for users to provide explicit feedback on other users nor to fill long questionnaires. In this way an accurate representation of the item selection process can be obtained, leading to the generation of better recommendations. Two in silico experiments have been performed to illustrate the operational steps of the proposed model and to demonstrate how the estimated utility is affected by interpersonal trust.

The paper is organized as follows: Section 2 presents related work on GRS and trust-based RS as well as background concepts on group recommendation and fuzzy-based GDM; Section 3 defines a fuzzy-based GDM model for group recommendations; Section 4 integrates, in the defined model, social factors based on user personality and interpersonal trust. Section 5 presents the results of an in-silico simulation of the proposed approaches to illustrate their properties. Conclusions are pointed out in Section 6.

\section{Background and Related Works}

Most work on RS focuses on the recommendation of items to individual users. GRSs go one step further and generate recommendations for items that are consumed by groups. Section 2.1 summarizes related works and illustrates the advancements carried out by the proposed model with respect to the state of the art; Section 2.2 introduces the group recommendation problem and describes existing approaches to RS and GRS; Section 2.3 defines background concepts on fuzzy-based GDM useful to appreciate the models described in the subsequent sections.

\subsection{Related Works}

Several GRSs have been proposed so far in the literature. Among the first systems there is MusicFX [12] that selects background music to be played in a fitness center to suit the group of people expected to exercise at a given time. User profiles are generated via an interview and music selection is based on profile aggregation. Another music recommendation system is Flytrap [13], which generates profiles from the music people listen to on their own computers whose presence in a room is identified by RFID tags.

Polylens [14] is a movies recommendation system that allows users to create groups and ask for recommendations that are built by aggregating individual recommendations (generated using users' star ratings). In the field of TV shows, Yu's TV [15] recommends television programs for families based on individual preferences for program features. Family Interactive TV [16] also filters television programs according to the viewers' preferences and uses implicit relevance feedback assessed through the actual program the viewer has chosen for watching. In [17] a prototype GRS for the popular Netflix media streaming service is also proposed.

Travel Decision Forum [18] assists a group of people to agree on the desired attributes of a planned joint holiday. Users indicate their preferences on a set of features (room facilities, sightseeing attractions, etc.) which are aggregated and a mediator agent supports the group to reach consensus. The Collaborative Advisory Travel System [19] is a similar system that induces profiles from user feedback on holiday packages. The Pocket Restaurant Finder [20] generates restaurant recommendations for groups of people based on preferences on cuisine type, restaurant amenities, price category, etc. Intrigue [21] is a GRS for touristic places which builds recommendations based on the aggregation of individual preferences.

The preceding systems have in common that the group recommendations take users' individual preferences into account without considering either the users' personality or the relationships among group members. Despite that in real contexts such aspects are crucial in the item selection process, systems addressing them have been introduced only recently. For example, in [7] a 30-questions test is used to determine a value representing how selfish or cooperative an user is in conflict situations. The obtained value is used, in turn, to weight the preferences of group members during aggregation. 
In [22] it has been pointed out that people tend to rely more on recommendations coming from people they trust than on anonymous ratings coming from similar users. According to [7], this is even more important when users have to decide on items to be consumed by a group. FilmTrust [23] is an example of trust-aware RS, which builds a network of trust among users based on explicit feedback. Users are asked to provide a trust rating for each person they add as a friend. Then, unknown items for each user are rated according to the average rating of trusted friends weighted by the value of trust. Another example is Epinions ${ }^{1}$, an e-commerce site that maintains a network of trust by asking users to indicate which members they trust or distrust. If no direct connections from an user to a rater exist for an item, trust propagation and aggregation algorithms are used to estimate indirect trust values [24].

A drawback of such personality and trust-based approaches is that they requires explicit feedback from users. To overcome this issue, a promising alternative is to build networks of trust from implicit information commonly shared on-line by users, e.g. data contained in social networks. According to [25], the complete transparency of this process compensates the fact that the trust networks obtained in such way are less accurate than those obtained with explicit feedback. For example, in [7], interpersonal trust has been estimated as a combination of 10 factors measured on Facebook profiles.

As an evolution of these latter approaches, the novel GRS model proposed in this paper combines both interpersonal trust and personality concepts in that of social influence. This is motivated by the fact that items selection in a group usually follows an argumentation process, where each member defends their own preferences and contradicts other's. In this process, interpersonal influence (dependent of trust and personality) is a major factor affecting opinion change toward a common decision. Taking such factors into account allows to define a more accurate representation of the reality, potentially leading to better recommendations.

Moreover, the proposed model introduces a GDM based approach to solve a GRS problem. In fact, while items selection for individual consumption can be considered as an interaction-free process, so manageable with standard RS techniques, when users interaction are expected to find an agreement among different hypotheses, a GDM problem can be outlined and specific techniques taking social influence into account can be applied. Being group recommendations generated from individual RS predictions (rather than from explicit preference statements), fuzzy-based approaches [26, 27, 28], which are intrinsically able to deal with uncertainty and inaccuracy of such predictions, have been preferred.

The application of GDM to GRS is a relatively new research area. To the best of our knowledge, few works have been published in this area and mainly related to consensus-reaching among group members. For example, in [29] a RS is used to generate individual recommendations for a group of users and a GDM consensus model is applied to update the preferences of the most discordant members making them as concordant as possible to average preferences. In [30] the same approach is applied to restaurant recommendations. With respect to such works, the model proposed in this paper applies a full GDM process that integrates social aspects of influence, trust and personality.

\subsection{Background on Group Recommendation}

A formal definition of the recommendation problem is the following: let $U=\left\{u_{1}, \ldots, u_{m}\right\}$ be a set of users, $X=\left\{x_{1}, \ldots, x_{n}\right\}$ a set of items that can be recommended, $R$ a totally ordered set whose values represent the utility of an item for a user (e.g. integers between 1 and 5 or real numbers between 0 and 1) and $f: U \times X \rightarrow R$ a utility function measuring how an item $x \in X$ is useful for a user $u \in U$; the purpose of a RS is to recommend, to each user $u$, the item $x^{*}$ that maximizes the utility function [1]:

\footnotetext{
${ }^{1}$ http://www.epinions.com/
} 


$$
x^{*}=\underset{x \in X}{\operatorname{argmax}} f(u, x)
$$

The central issue of RSs is that $f$ is not completely defined over the space $U \times X$. Indeed, in typical applications, a user never expresses preferences on each available item. A RS should also be able to estimate the values of the utility function in the space of data where it is not defined, extrapolating from the points of $U \times X$ where it is known. In other words, the goal is to predict the rating that a user would give to an unknown item.

Several techniques exist to predict unknown ratings. In content-based approaches [31], the utility $f(u, x)$ of an unknown item $x$ for user $u$ is predicted by considering defined values of $f$ for items that are considered similar to $x$. In collaborative approaches [32], unknown ratings are estimated from those made available by other users who have evaluated the same items in a similar way to user $u$ and are therefore assumed to have similar tastes. In model-based approaches [33] the history of the RS in not directly used to make predictions but to learn a model that is then used to generate recommendations.

In some cases the recommended items are consumed in groups rather than individually so provided recommendations should fit the preferences of different group members at the same time. GRS specifically address this issue by aggregating information about individual group members generated by standard RSs. Several group recommendation strategies and methods have been defined so far by different researchers [5]. Recommendations aggregation strategies generate recommendations for group members through a standard RS. Then, the individual lists of recommended items are used to build a unique list for the group as a whole. On the other hand, preferences aggregation strategies combine users' preferences in order to obtain recommendations for the whole group with a standard algorithm. In this way the group is seen as a pseudo-user reflecting the interests of all members [34].

In both strategies, the implemented aggregation method plays a key role [6, 35]. For example, the average method estimates the group utility of an item by mediating the individual utility of that item for any group member. Denoting $U_{G} \subseteq U$ the set of users belonging to a group $G$ and $X_{G} \subseteq X$ the set of all items recommended to at least one member of $G$, the group utility of an item $x$ is estimated as follows:

$$
f\left(U_{G}, x\right)=\frac{\sum_{u \in U_{G}} f(u, x)}{\# U_{G}}
$$

In case of preferences aggregation, equation (2) is calculated for every $x \in X$, while in case of recommendations aggregation it is calculated just for $x \in X_{G}$. In both cases, the elements with highest group utility are recommended to the group $G$.

The average without misery method looks for the optimal decision for the group $G$, without making any of the members really unhappy with such decision. So, any item $x \in X$ (or $x \in X_{G}$ in case of recommendations aggregation) with $f(u, x)$ below a given threshold for at least one user $u \in U_{G}$ receives a penalty in the calculation of the group utility value (or it is simply removed from the list of group recommendations). The least misery method aims at minimizing the overall misery of the group by considering the minimum individual utility as the group utility for each item. Conversely, the most pleasure method maximizes the overall pleasure by considering the maximum individual utility as the group utility.

The multiplicative method obtains the group utility of any item $x \in X$ (or $x \in X_{G}$ in case of recommendations aggregation) by multiplying the individual estimated utilities $f(u, x)$ of $x$ for any group member $u \in U_{G}$ : 


$$
f\left(U_{G}, x\right)=\prod_{u \in U_{G}} f(u, x)
$$

Such method performs well when utility values are expressed with values greater than 1 (e.g. with star rating) giving more importance to higher utilities rather than to lower ones. In [6] it was shown that the recommendations provided with such method (within a recommendations aggregation strategy) are the most positively accepted by users, followed by those provided by the average, average without misery and most pleasure methods, respectively.

\subsection{Background on Fuzzy Group Decision Making}

The group recommendation problem, as formalized in Section 2.2, can be seen as a special case of the GDM problem. In GDM, a group of experts evaluate a set of alternatives with the aim of selecting the best one for the group. To this end, each expert expresses preferences on alternatives, which are aggregated to arrive at a collective preference degree on each alternative from which a ranking of alternatives is generated $[36,37,38,39]$.

Similarly, the aim of GRSs, is to select, from a given catalogue, the item or the set of items that fit the preferences of all (or the majority of) users belonging to a group. Differently from GDM, users do not need to explicitly state their preferences on items but an utility function, estimating such preferences, is already available as output of a standard RS algorithm. As in GDM, user preferences must be aggregated, the collective preference degree of each item must be calculated and a ranking of items must be generated. It is important to consider that GDM problems, instantiated in this way, consist of a decision process based on predicted utility values rather than on explicit preference statements collected among experts. This suggests to rely on approaches that are intrinsically able to deal with the uncertainty resulting from prediction inaccuracy, like those based on fuzzy sets $[40,41$, 42, 43].

A GDM problem is characterized by a group of experts $E=\left\{e_{1}, \ldots, e_{m}\right\}$ that express their preferences on a finite set of alternatives $X=\left\{x_{1}, \ldots, x_{n}\right\}$ to reach a common solution. Several ways to model experts' preferences exist. Among them, the Fuzzy Preference Relation (FPR) is one of the most commonly used [44]. It specifies the degree to which each alternative $x_{i} \in X$ is at least as good as any other alternative. Formally, a FPR is a fuzzy relation $P$, i.e. a fuzzy set on $X \times X$ with a membership function $\mu_{P}: X \times X \rightarrow[0,1]$ such that:

$$
\mu_{P}\left(x_{i}, x_{j}\right)= \begin{cases}1 & \text { if } x_{i} \text { is definitely preferred to } x_{j}, \\ a \in(0.5,1) & \text { if } x_{i} \text { is slightly preferred to } x_{j}, \\ 0.5 & \text { if } x_{i} \text { and } x_{j} \text { are equally preferred, } \\ b \in(0,0.5) & \text { if } x_{j} \text { is slightly preferred to } x_{i} \\ 0 & \text { if } x_{j} \text { is definitely preferred to } x_{i} .\end{cases}
$$

A FPR can be conveniently represented as a $n \times n$ matrix $P=\left(p_{i j}\right)$ where $p_{i j}=\mu_{P}\left(x_{i}, x_{j}\right)$.

Once experts have expressed preferences on the set of alternatives, $m$ individual FPRs $P_{1}, \ldots, P_{m}$ are available where $P_{k}=\left(p_{i j}^{k}\right)$. The first step to carry out to reach a decision is the fusion of the individual FPRs into a collective one using an aggregation operator. Several operators have been proposed for this purpose. Among them, the Ordered Weighted Average (OWA) [45] is one of the most commonly used because it allows for the implementation of both importance degrees and the concept of majority. It is defined as follows: 


$$
O W A\left(p_{i j}^{1}, \ldots, p_{i j}^{m}\right)=\sum_{k=1}^{m} w_{k} p_{i j}^{\sigma(k)}
$$

where $w_{1}, \ldots, w_{m} \in[0,1]$ are weights such that $\sum_{k=1}^{m} w_{k}=1$ while $\sigma:\{1, \ldots m\} \rightarrow\{1, \ldots m\}$ is a permutation function such that $p_{i j}^{\sigma(k)} \geq p_{i j}^{\sigma(k+1)}$ for $k \in\{1, \ldots, m-1\}$.

The behavior of OWA strictly depends on its weighting vector. In [26], the authors propose to initialize weights starting from a non-decreasing proportional linguistic quantifier. Fuzzy quantifiers are defined in [46] as imprecise representations of the amount of items satisfying a given predicate. A proportional fuzzy quantifier $Q$ is as fuzzy subset of the unit interval $[0,1]$ where, for any $x \in[0,1]$, $\mu_{Q}(x)$ represents the degree to which the proportion $x$ is compatible with the meaning of the quantifier. A non-decreasing fuzzy quantifier satisfies the additional monotonicity property $\mu_{Q}\left(x_{1}\right) \geq$ $\mu_{Q}\left(x_{2}\right)$ if $x_{1}>x_{2}$. The usual membership function of a non-decreasing proportional fuzzy quantifier is defined as:

$$
\mu_{Q}(r)= \begin{cases}0 & \text { if } r<a, \\ \frac{r-a}{b-a} & \text { if } a \leq r \leq b, \\ 1 & \text { if } r>b .\end{cases}
$$

with $a, b, r \in[0,1]$. Examples of non-decreasing proportional linguistic quantifiers and the related membership functions are shown in Figure $1[47,48]$.
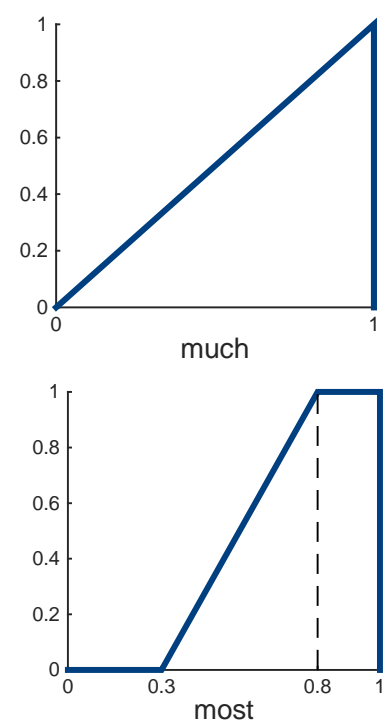

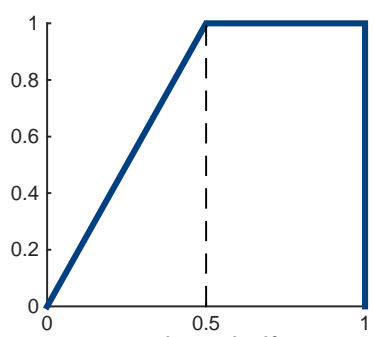

at least half

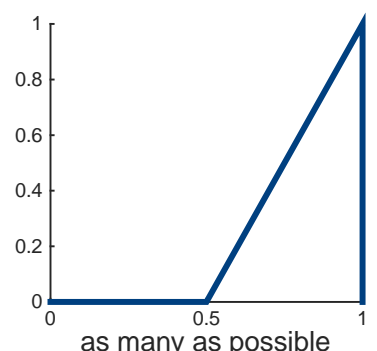

Figure 1. Example of non-decreasing proportional linguistic quantifiers

The weights of an OWA operator of dimension $m$ can be obtained from a non-decreasing proportional linguistic quantifier as follows [45]:

$$
w_{k}=\mu_{Q}\left(\frac{k}{m}\right)-\mu_{Q}\left(\frac{k-1}{m}\right) ; k \in\{1, \ldots, m\} .
$$

where $Q$ must be selected to reflect the fusion strategy that the decision makers would apply (i.e. the ratio of experts that are expected to be satisfied with the aggregated value). 
Once the collective FPR $P$ is obtained, the alternatives are rated by associating a degree of preference $\phi\left(x_{i}\right)$ to each $x_{i} \in X$ based on $P$. Also, in this case, several measures are possible. In [26, 49] the Quantifier Guided Dominance Degree (QGDD) has been proposed to calculate the dominance that one alternative has over all the others in a soft majority sense:

$$
\phi_{Q G D D}\left(x_{i}\right)=O W A_{Q}\left(p_{i j} ; j=1, \ldots, n ; j \neq i\right)
$$

where $O W A_{Q}$ indicates the OWA operator whose weights are derived using a non-decreasing proportional linguistic quantifier representing the concept of soft majority to implement. After having rated the available alternatives, the one with the highest degree of preference is the solution of the GDM problem.

\section{A GDM Model for Group Recommendation}

Let $X_{u}$ be the recommendation list built for user $u \in U$ using a standard RS, $U_{G} \subseteq U$ a group of users and $X_{G}=\bigcup_{u \in U_{G}} X_{u}$ the set of items recommended to the group members. Clearly, interpreting $U_{G}$ as a set of experts and $X_{G}$ as a set of alternatives, we can turn a group recommendation problem into a GDM one. Then, the estimated utility $f(u, x)$, with $x \in X_{G}$ and $u \in U_{G}$, can be seen as an estimation of experts' preferences.

Nevertheless, to apply the fuzzy GDM model described in Section 2.3, user preferences must be expressed in term of FPRs. According to [41,50] $[26,41]$, utility values $\left(r_{1}, \ldots, r_{n}\right)$, normalized in $[0,1]$, can be transformed into a FPR by any function $H:[0,1] \times[0,1] \rightarrow[0,1]$ verifying the following conditions:

- $H(x, y)+H(y, x)=1 \forall x, y \in[0,1]$

- $H(x, x)=0.5 \forall x \in[0,1]$;

- $H(x, 0)=1 \forall x \in(0,1]$;

- $H(x, y)>0.5 \forall x \in x>y$.

According to [41], a feasible utility-to-FPR transformation function respecting the required conditions is $H(x, y)=(1+x-y) / 2$. Let $f: U_{G} \times X_{G} \rightarrow[0,1]$ be the RS-estimated utility function, with $U_{G}=\left\{u_{1}^{G}, \ldots, u_{m}^{G}\right\} \subseteq U$ and $X_{G}=\left\{x_{1}^{G}, \ldots, x_{n}^{G}\right\} \subseteq X$, thus, the FPR $P_{k}=\left(p_{i j}^{k}\right)$ corresponding to a $u_{k}^{G} \in U_{G}$ can be defined according to the following equation:

$$
p_{i j}^{k}=\frac{1+f\left(u_{k}^{G}, x_{i}^{G}\right)-f\left(u_{k}^{G}, x_{j}^{G}\right)}{2} .
$$

Once all individual FPRs $P_{k}$ with $k \in\{1, \ldots, m\}$ are obtained, they are aggregated to derive the collective FPR $P$ with the OWA operator, according to equations (5)-(7). Then, it is possible to calculate the group preference $\phi\left(x_{i}^{G}\right)$ for each item $x_{i}^{G} \in X_{G}$ with the QGDD measure as per equation (8). The global ranking of the items results by ordering them decreasingly based on their group preference degree and the top-ranked elements are recommended to the group. In addition, an estimation of the group utility of each item $x \in X_{G}$ can be obtained through normalization as follows:

$$
f\left(U_{G}, x\right)=\frac{\left(\phi(x)-\phi_{\min }\right) \cdot\left(f_{\max }-f_{\min }\right)}{\left(\phi_{\max }-\phi_{\min }\right)}+f_{\min }
$$

where $\phi_{\min }$ and $\phi_{\max }$ are, respectively, the minimum and maximum values of the group preference $\phi\left(x_{i}^{G}\right)$ for $i \in\{1, \ldots, n\}$, while $f_{\min }$ and $f_{\max }$ are the minimum and maximum of the utility function $f\left(u_{k}^{G}, x_{i}^{G}\right)$ for $k \in\{1, \ldots, m\}$ and $i \in\{1, \ldots, n\}$. 
An important aspect to take into account in the FPR aggregation process is the choice of the linguistic quantifier guiding the OWA operator. For example, let $U_{G}=\left\{u_{1}^{G}, u_{2}^{G}, u_{3}^{G}, u_{4}^{G}\right\}$ be the users belonging to a group $G$ and $X_{G}=\left\{x_{1}^{G}, x_{2}^{G}, x_{3}^{G}, x_{4}^{G}\right\}$ the items recommended to at least a group member. Let assume that the estimated individual utility for each group member is as reported in Table 1. Using the proposed GDM model to build group recommendations, different results are obtained according to the chosen quantifier. Table 2 summarizes the results obtained using different linguistic quantifiers, compared with results obtained with standard recommendations aggregators as seen in Section 2.2.

\begin{tabular}{c|cccc}
\hline \hline \multirow{2}{*}{ Group members } & \multicolumn{4}{|c}{ Individual item utilities } \\
\cline { 2 - 5 } & $x_{1}^{G}$ & $x_{2}^{G}$ & $x_{3}^{G}$ & $x_{4}^{G}$ \\
\hline$u_{1}^{G}$ & 1.00 & 0.60 & 0.80 & 0.20 \\
$u_{2}^{G}$ & 0.60 & 0.60 & 0.80 & 0.20 \\
$u_{3}^{G}$ & 0.50 & 0.60 & 0.40 & 1.00 \\
$u_{4}^{G}$ & 0.30 & 0.60 & 0.40 & 1.00 \\
\hline \hline
\end{tabular}

Table 1. Sample individual item utilities.

As shown in Table 2, the "average" aggregator assigns the same importance to all individual utilities. The "average without misery" aggregator has a similar behavior but excludes items $x_{1}^{G}$ and $x_{4}^{G}$ because their utility is too low for some users $\left(u_{1}^{G}\right.$ and $\left.u_{2}^{G}\right)$. The "least misery" and "most pleasure" aggregators use, in turn, the lower and the higher individual utility of each item, while the "multiplication" aggregator, privileges lower individual utilities over higher ones (being utilities between 0 and 1 ).

\begin{tabular}{l|cccc}
\hline \multirow{2}{*}{ Aggregators } & \multicolumn{4}{|c}{ Ranked group utilities } \\
\cline { 2 - 6 } & $1^{\text {st }}$ & $2^{\text {nd }}$ & $3^{\text {rd }}$ & $4^{\text {th }}$ \\
\hline average & $x_{1}^{G}(0.60)$ & $x_{2}^{G}(0.60)$ & $x_{3}^{G}(0.60)$ & $x_{4}^{G}(0.60)$ \\
average without misery & $x_{2}^{G}(0.60)$ & $x_{3}^{G}(0.60)$ & $x_{1}^{G}(0.00)$ & $x_{4}^{G}(0.00)$ \\
least misery & $x_{2}^{G}(0.60)$ & $x_{3}^{G}(0.40)$ & $x_{1}^{G}(0.30)$ & $x_{4}^{G}(0.20)$ \\
most pleasure & $x_{1}^{G}(1.00)$ & $x_{4}^{G}(1.00)$ & $x_{3}^{G}(0.80)$ & $x_{2}^{G}(0.60)$ \\
multiplication & $x_{2}^{G}(0.13)$ & $x_{3}^{G}(0.10)$ & $x_{1}^{G}(0.09)$ & $x_{4}^{G}(0.04)$ \\
\hline at least half (GDM) & $x_{4}^{G}(1.00)$ & $x_{1}^{G}(0.35)$ & $x_{3}^{G}(0.35)$ & $x_{2}^{G}(0.20)$ \\
much (GDM) & $x_{1}^{G}(0.50)$ & $x_{2}^{G}(0.50)$ & $x_{3}^{G}(0.50)$ & $x_{4}^{G}(0.50)$ \\
most (GDM) & $x_{2}^{G}(1.00)$ & $x_{3}^{G}(0.65)$ & $x_{1}^{G}(0.25)$ & $x_{4}^{G}(0.20)$ \\
as many as possible (GDM) & $x_{2}^{G}(1.00)$ & $x_{1}^{G}(0.85)$ & $x_{3}^{G}(0.85)$ & $x_{4}^{G}(0.20)$ \\
\hline \hline
\end{tabular}

Table 2. Ranked group utilities obtained using different aggregators

Similarly, by adopting the GDM-based model, different behaviors are obtained by choosing different quantifiers for the OWA operator. Given that FPRs are built comparing, for each user, the individual utilities of different items, the GDM model operates on relative utilities rather than on absolute ones. By taking that into account, it can be observed in Table 2 that the "much" quantifier behaves like the "average" operator. The "at least half" privileges relative utilities associated to most enthusiastic users, so $x_{4}^{G}$ wins thanks to its high estimated relative utility for users $u_{3}^{G}$ and $u_{4}^{G}$. Conversely, the "as many as possible" quantifier privileges relative utilities associated to least enthusiastic users so $x_{2}^{G}$ wins thanks to its high estimated relative utility for users $u_{1}^{G}$ and $u_{3}^{G}$. Finally, 
the "most" quantifier privileges relative utilities associated to lower-intermediate users so, in this case, the winner is $x_{2}^{G}$.

The previous example demonstrates the flexibility of the proposed approach even without the introduction of social influence (as explained in the next section) and motivates the choice of the OWA operator for preferences aggregation. In fact, it allows to design different aggregation strategies by simply selecting different linguistic quantifiers. Moreover, when needed, a new strategy can be introduced by designing a new quantifier.

\section{Influence-Based Recommendations}

When selecting an item for consumption by a group of users, the final choice is often deeply affected by the personality of the group members. In fact, due to interpersonal influence, individual preferences may change during the process when information and opinions are exchanged in social interactions. In order to take social influence into account, we propose an improved GDM-based model for group recommendations that integrates social elements in accordance with the approach proposed in [9].

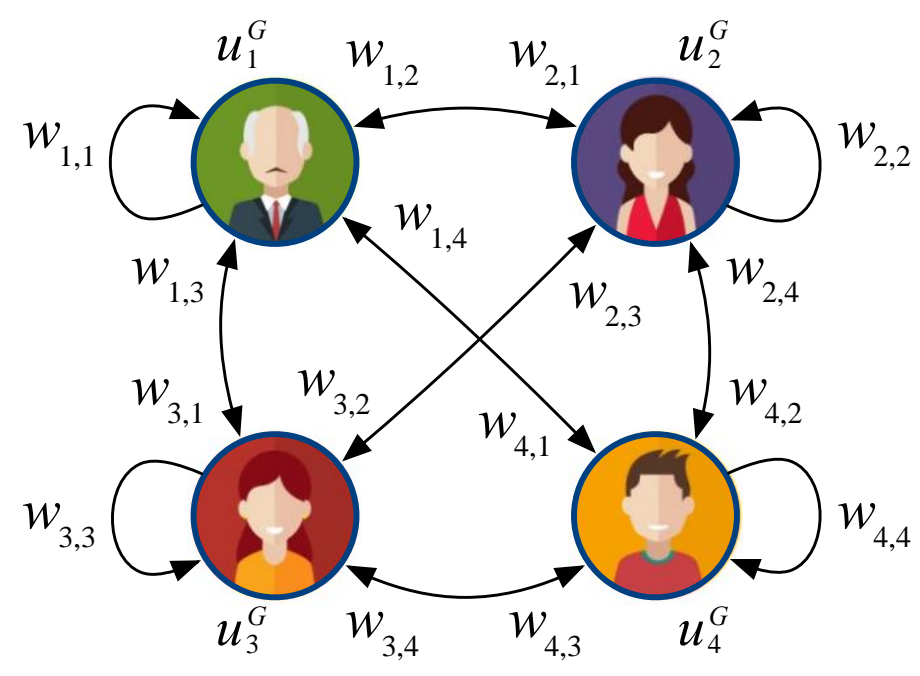

Figure 2. A sample SIN with 4 group members

In particular, the configuration and strength of social influence among group members is evaluated based on interpersonal trust and represented with a Social Influence Network (SIN). In analogy with the definition provided in $[51,52]$, a SIN is a directed graph associating, each pair of group members $\left(u_{i}^{G}, u_{j}^{G}\right) \in U_{G}^{2}$ a weight $w_{i j} \in[0,1]$ that measures the strength of the influence of the $j$-th member on the $i$-th one (see Figure 2). A SIN involving users from $U_{G}$ can be summarized by an $m \times m$ fuzzy adjacency matrix $W=\left(w_{i j}\right)$ where selected weights verify the normalization property:

$$
\sum_{j=1}^{m} w_{i j}=1 \forall i \in\{1, \ldots, m\}
$$

SIN weights can be settled according to explicit trust statements, like in [9], or inferred by analyzing social interactions among group members, e.g. by looking at implicit information contained in social networks, like Facebook or Twitter. Section 4.1 describes different methods that can be used to build such SIN. The obtained SIN, in its turn, is used to complete user preferences in case of missing elements (e.g. due to limited coverage of the underlying RS) and to evolve them toward a shared set of preferences that is then used to build recommendations. Such process is described in section 4.2. 


\subsection{Social Influence Estimation}

In [53] it was demonstrated that trust and tie strength are conceptually different but strongly correlated. In [54], 74 Facebook variables have been identified as potential predictors of tie strength. By relying on these results, in [7], interpersonal trust has been estimated as a linear combination of 10 factors measured on Facebook profiles, although a reliable estimation of trust strength can be obtained by just considering, for each $u_{i}^{G}, u_{j}^{G} \in U_{G}$ with $i \neq j$, the following 5 factors:

- $f_{1}\left(u_{i}^{G}, u_{j}^{G}\right)$ represents the amount of common friends between $u_{i}^{G}$ and $u_{j}^{G}$, ranging from 0.1 (less than 5) to 1 (more than 25);

- $f_{2}\left(u_{i}^{G}, u_{j}^{G}\right)$ is the percentage of pictures where $u_{i}^{G}$ and $u_{j}^{G}$ appear together over the total number of pictures in the $u_{i}^{G}$ profile;

- $f_{3}\left(u_{i}^{G}, u_{j}^{G}\right)$ is the duration of the relationship between $u_{i}^{G}$ and $u_{j}^{G}$ ranging from 0.1 (less than 1 year) to 1 (more than 10 years), obtained comparing information on age, schools, universities, work and family relations;

- $f_{4}\left(u_{i}^{G}, u_{j}^{G}\right)$ is the percentage of common interests described in the profiles of $u_{i}^{G}$ and $u_{j}^{G}$ (movies, books, joined groups, etc.) over the total number of interests declared in the $u_{i}^{G}$ profile;

- $f_{5}\left(u_{i}^{G}, u_{j}^{G}\right)$ is the strength of the declared status between $u_{i}^{G}$ and $u_{j}^{G}$ ranging from 0.1 (barely know) to 1 (couple).

The trust level of any group member $u_{i}^{G}$ on any other member $u_{j}^{G}$ can be then obtained as the following weighted sum:

$$
\operatorname{trust}\left(u_{i}^{G}, u_{j}^{G}\right)=\sum_{k=1}^{5} w_{k} f_{k}\left(u_{i}^{G}, u_{j}^{G}\right)
$$

where $i \neq j$ and the weights $w_{k}$ with $k \in\{1, \ldots, 5\}$ are chosen so that $\sum_{k=1}^{5} w_{k}=1$. According to [7], a feasible set of weights is: $w_{1}=0.4 ; w_{2}=w_{3}=0.2 ; w_{4}=0.15$ and $w_{5}=0.05$.

Once the interpersonal trust among group members is estimated, to build a SIN, building the SIN still requires the estimation of users' self-confidence which measures the attitude of users to remain faithful to their initial preferences, mitigating the effects of social influence. In [7], a similar attribute is estimated based on the Thomas-Kilmann Conflict Mode Instrument (TKI), a test made of 30 questions with two possible answers [55].

TKI defines five personality modes when dealing with conflicts: competing, collaborating, avoiding, accommodating and compromising. Depending on the answers provided to test questions, a score is assigned to each personality mode. Then, the obtained results are summarized along two basic dimensions: assertiveness and cooperativeness through a weighted sum of the obtained scores. Given the assertiveness $a\left(u_{i}^{G}\right)$ and the cooperativeness $c\left(u_{i}^{G}\right)$ of user $u_{i}^{G} \in U_{G}$ and assuming that both values are defined in $[0,1]$, the self-confidence of $u_{i}^{G}$ can be obtained as follows:

$$
\operatorname{self}\left(u_{i}^{G}\right)=\frac{1+a\left(u_{i}^{G}\right)-c\left(u_{i}^{G}\right)}{2}
$$

A drawback of this approach is that it requires that group members fill a 30-questions test before start using the system. Nevertheless, some studies $[56,57]$ correlate conflict management styles with the so-called five-factors personality traits (extraversion, agreeableness, neuroticism, conscientiousness and openness). Based on these studies, if the personality traits are known, it is possible to estimate the levels of assertiveness and cooperativeness of a given user and, subsequently via equation (13), the self-confidence as well. 
Several tests exist to estimate personality factors, examples of which are the Five-Factor Personality Inventory or the Revised NEO Personality Inventory [58]. Unfortunately, such approaches suffer from the same limitation discussed above, i.e. users are required to complete long questionnaires before using the system. Nevertheless, some approaches exist to predict personality directly from the language used in social media. For example, in [59], an algorithm for the prediction of the five-factors traits from the textual analysis of users' Facebook status updates is defined. Moreover, in a similar work, an on-line tool for personality prediction from Facebook named Apply Magic Sauce ${ }^{2}$ has been implemented [60].

Once $\operatorname{self}\left(u_{i}^{G}\right)$ and $\operatorname{trust}\left(u_{i}^{G}, u_{j}^{G}\right)$ are estimated for $i, j \in\{1, \ldots, m\}$, it is possible to obtain SIN weights as follows:

$$
w_{i j}= \begin{cases}\left(1-\operatorname{self}\left(u_{i}^{G}\right)\right) \cdot \frac{\operatorname{trust}\left(u_{i}^{G}, u_{j}^{G}\right)}{\sum_{k \in\{1, \ldots, m\} \backslash i} \operatorname{trust}\left(u_{i}^{G}, u_{k}^{G}\right)} & \text { if } i \neq j, \\ \operatorname{self}\left(u_{i}^{G}\right) & \text { if } i=j .\end{cases}
$$

When $\operatorname{trust}\left(u_{i}^{G}, u_{j}^{G}\right)=O \forall j \in\{1, \ldots, m\} \backslash i$ (i.e. when a user does not trust any other user) is managed by setting $w_{i i}=1$ and $w_{i j}=0 \forall j \in\{1, \ldots, m\} \backslash i$. It is trivial to demonstrate that the obtained matrix $W$ fulfills the normalization property (11).

\subsection{Social Influence Application}

The prediction accuracy of a RS usually improves when the amount of available data increases $[1,5]$. In real cases, due to lack of data, it can be not possible for a RS to estimate the utility of some items for some users. Indeed, the prediction coverage of a RS represents the percentage of items for which a recommendation can be generated while the user-space coverage is the percentage of users for which at least one recommendation can be generated [61]. Due to lack of coverage, the RS-estimated utility function may remain undefined for some pair $(u, x)$ with $u \in U_{G}$ and $x \in X_{G}$. In such cases the FPRs generated with equation (9) may have missing values.

According to [9], it is possible to estimate a missing preference $p_{i j}^{k}$ of a given FPR $P_{k}$, with $k \in$ $\{1, \ldots, m\}$ and $i, j \in\{1, \ldots, n\}$, by aggregating the corresponding FPR values of influencing group members, weighted by the influence strength (described in the SIN). This method simulates the human process of opinion forming about unfamiliar alternatives by surveying the opinion of trusted people. The aggregation is performed through the Importance-Induced OWA (I-IOWA) operator, defined in [62]. Let $\left(p_{i j}^{1}, \ldots, p_{i j}^{m}\right)$ be a list of preference values coming from other group members and $w_{k 1}, \ldots, w_{k m}$ the SIN weights modelling the influence of users on $u_{k}$, the missing preference $p_{i j}^{k}$ can be estimated as follows:

$$
\varepsilon\left(p_{i j}^{k}\right)=I-I O W A_{Q}\left(\left(p_{i j}^{1}, w_{k 1}\right), \ldots,\left(p_{i j}^{m}, w_{k m}\right)\right)=\sum_{l=1}^{m} u_{k l} p_{i j}^{\sigma_{k}(l)}
$$

where $\sigma_{k}:\{1, \ldots m\} \rightarrow\{1, \ldots m\}$ is a permutation function such that $w_{k \sigma(l)} \geq w_{k \sigma(l+1)}$ while, similarly to equation (7), the parameters $u_{k l}$, for $l \in\{1, \ldots, m\}$, are obtained as follows:

$$
u_{k l}=\mu_{Q}\left(\frac{S_{k}(l)}{S_{k}(m)}\right)-\mu_{Q}\left(\frac{S_{k}(l-1)}{S_{k}(m)}\right)
$$

\footnotetext{
2 https://applymagicsauce.com/
} 
where $Q$ is a non-decreasing proportional fuzzy quantifier defined by the membership function $\mu_{Q}$ and $S_{k}(l)=\sum_{h=1}^{l} w_{k \sigma(h)}$.

Undefined FPR elements of group members are not considered in equation (15). If some preference value is still missing after the application of the preceding equations (e.g. when the same preferences are missing in all the FPRs of trusted experts), then the estimation process is repeated on FPRs injected with estimated values. The process is iterated until no additional values can be calculated. Moreover, to make injected values as consistent as possible with the existing ones, in [63] it is suggested to refine them iteratively as follows:

$$
\varepsilon\left(p_{i j}^{k}\right)=\frac{\sum_{l=1}^{n}\left(\varepsilon_{l}^{1}\left(p_{i j}^{k}\right)+\varepsilon_{l}^{2}\left(p_{i j}^{k}\right)+\varepsilon_{l}^{3}\left(p_{i j}^{k}\right)\right)}{3 n}
$$

where $\varepsilon_{l}^{1}\left(p_{i j}^{k}\right), \varepsilon_{l}^{2}\left(p_{i j}^{k}\right)$ and $\varepsilon_{l}^{3}\left(p_{i j}^{k}\right)$ (with $l \in\{1, \ldots, n\}$ ) are different estimators of $p_{i j}^{k}$ based on defined preferences values of the same FPR that are obtained as:

$$
\varepsilon_{k}^{1}\left(p_{i j}^{k}\right)=p_{i l}^{k}+p_{l j}^{k}-0.5 ; \varepsilon_{k}^{2}\left(p_{i j}^{k}\right)=p_{l j}^{k}-p_{l i}^{k}+0.5 ; \varepsilon_{k}^{3}\left(p_{i j}^{k}\right)=p_{i l}^{k}-p_{j l}^{k}+0.5
$$

As the initial estimation, the refinement of estimated values through equations (17)-(18) is done in several iterations until no additional values can be calculated.

To simulate the effects of social influence between group members, the individual FPRs generated with equation (9), and completed according to equations (15)-(18) if needed, are evolved using the SIN. To do that, an iterative process is applied where, at each step, each individual FPR is slightly changed to take into account the influence of trusted members. This is done again with equations (15)(16) applied to all FPR values rather than just on undefined ones. Being $P_{k}^{(1)}$ the initial FPR of the $k$-th user, by extending equation (15) to matrices, the $k$-th FPR after $t$ steps is obtained as:

$$
P_{k}^{(t)}=I-I O W A_{Q}\left(\left(P_{1}^{(t-1)}, w_{k 1}\right), \ldots,\left(P_{m}^{(t-1)}, w_{k m}\right)\right)
$$

In practical applications the preferences evolution may be stopped after a fixed number of iterations or when the average absolute difference between FPRs values of two successive steps is under a given threshold $\theta$ i.e. when:

$$
\frac{1}{m \cdot n^{2}} \sum_{1 \leq i, j \leq n ; 1 \leq k \leq m}\left|p_{i j}^{k(t)}-p_{i j}^{k(t-1)}\right| \leq \theta
$$

In [9] it has been demonstrated that, under certain conditions, all FPRs $P_{k}^{(t)}$ for $k \in\{1, \ldots, m\}$ are expected to converge to the same FPR in a limited number of steps. Anyway, when the stopping conditions are met but there is no convergence, the obtained FPRs can be aggregated through the OWA operator defined in Section 2.3.

When the collective FPR is obtained in such way, the group preference $\phi\left(x_{i}^{G}\right)$ of each item $x_{i}^{G} \in$ $X_{G}$ is calculated as described in section 3 , the items ranking is computed and the top elements are recommended to the group. Optionally, an estimation of the group utility of each item $x \in X_{G}$ can be obtained through equation (10). 


\section{Experiments and Evaluation}

This section describes two in silico experiments of the proposed models aimed at illustrating their operational steps. The first experiment applies the GDM model for group recommendation defined in Section 3 to a concrete scenario while the second experiment also takes social influence into account by applying the extended model defined in Section 4. The intention is to show the differences between the two models and demonstrate how the estimated utility is affected by interpersonal trust.

Let $U_{G}=\left\{u_{123}, u_{335}, u_{467}\right\}$ be a subset of systems users belonging to a given group $G$ and $X_{123}=$ $\left\{x_{12}, x_{25}, x_{39}, x_{77}\right\}, X_{335}=\left\{x_{12}, x_{46}, x_{67}, x_{77}\right\}$ and $X_{467}=\left\{x_{39}, x_{46}, x_{77}, x_{89}\right\}$ the lists of items recommended to $U_{G}$ members by a standard RS. The set of items recommended to at least one group member is $X_{G}=X_{123} \cup X_{335} \cup X_{467}$. By assuming that the individual utilities for items in $X_{G}$ are those summarized in Table 3 (where - represents an undefined prediction), it is possible to build the FPRs associated to $U_{G}$ members according to equation (9), as summarized in Table 4. Given that the utilities of $x_{89}$ for $u_{123}$ and of $x_{67}$ for $u_{467}$ are unknown, the corresponding FPR values are estimated assuming indifference of preference between unrated items and other items (i.e. by setting the corresponding FPR values to 0.5 ) and iterating equations (17)-(18) until convergence to make injected values more consistent.

\begin{tabular}{c|ccccccc}
\hline \hline \multirow{2}{*}{ Group members } & \multicolumn{7}{|c}{ Individual item utilities } \\
\cline { 2 - 8 } & $x_{12}$ & $x_{25}$ & $x_{39}$ & $x_{46}$ & $x_{67}$ & $x_{77}$ & $x_{89}$ \\
\hline$u_{123}$ & 1.00 & 0.90 & 1.00 & 0.50 & 0.20 & 0.80 & - \\
$u_{335}$ & 0.90 & 0.30 & 0.60 & 1.00 & 0.90 & 1.00 & 0.10 \\
$u_{467}$ & 0.10 & 0.30 & 0.90 & 0.80 & - & 0.80 & 1.00 \\
\hline \hline
\end{tabular}

Table 3. Individual item utilities

By using equations (5)-(7) to aggregate users' FPRs with the OWA operator guided by the linguistic quantifier "much" (see Figure 1), the following collective FPR is obtained:

$$
P=\left(\begin{array}{lllllll}
0.50 & 0.58 & 0.42 & 0.45 & 0.55 & 0.40 & 0.52 \\
0.42 & 0.50 & 0.33 & 0.37 & 0.47 & 0.32 & 0.44 \\
0.58 & 0.67 & 0.50 & 0.53 & 0.62 & 0.48 & 0.60 \\
0.55 & 0.63 & 0.47 & 0.50 & 0.59 & 0.45 & 0.58 \\
0.45 & 0.53 & 0.38 & 0.41 & 0.50 & 0.36 & 0.51 \\
0.60 & 0.68 & 0.52 & 0.55 & 0.64 & 0.50 & 0.63 \\
0.48 & 0.56 & 0.40 & 0.42 & 0.49 & 0.37 & 0.50
\end{array}\right) .
$$

Then, it is possible to obtain the group preference of each item in terms of QGDD through equation (8) and, in turn, their group utility with equation (10), as summarized in the first row of Table 5. So, according to the standard GDM-based model, the top-ranked items for group consumption are: $x_{77}$, $x_{39}$ and $x_{46}$.

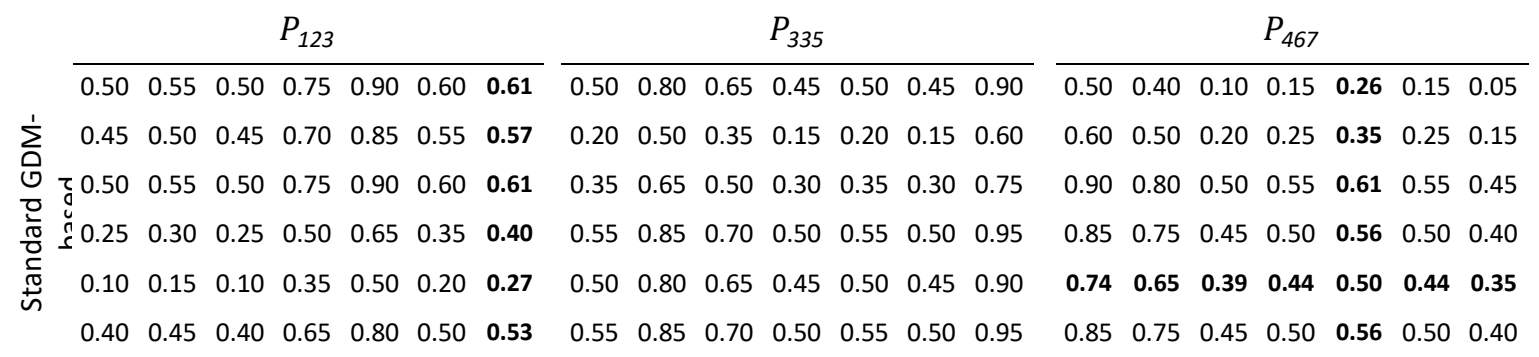




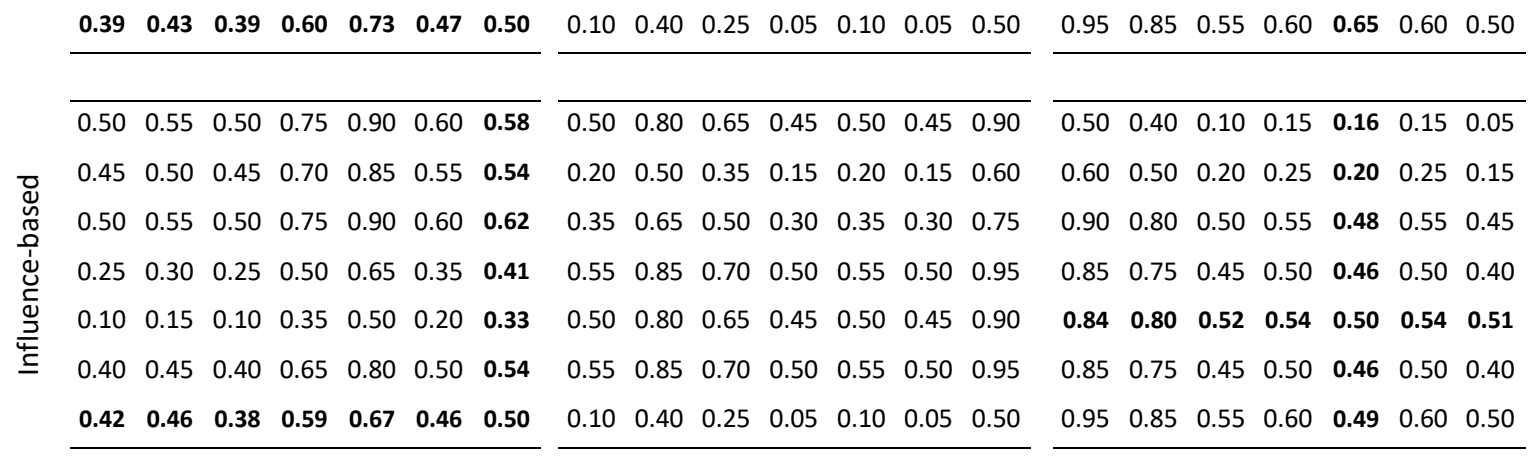

Table 4. Group user FPRs generated from individual item utilities in the two experiments, bold values correspond to initially unknown preferences

Now let us suppose that by applying one of the methods described in section 4.1 it is possible to estimate the following level of interpersonal trust between users: $\operatorname{trust}\left(u_{123}, u_{335}\right)=0.3$; $\operatorname{trust}\left(u_{335}, u_{123}\right)=0.8 ; \operatorname{trust}\left(u_{123}, u_{467}\right)=0.9 ; \operatorname{trust}\left(u_{467}, u_{123}\right)=0 ; \operatorname{trust}\left(u_{335}, u_{467}\right)=0.8$ and $\operatorname{trust}\left(u_{467}, u_{335}\right)=0.8$. Let us also assume the following levels of users' selfishness calculated through equation (13): $\operatorname{self}\left(u_{123}\right)=0.5 ; \operatorname{self}\left(u_{335}\right)=0.2$ and $\operatorname{self}\left(u_{467}\right)=0.8$; according to equation (14) it is possible to obtain the SIN represented by the following matrix:

$$
W=\left(\begin{array}{ccc}
0.5 & 0.12 & 0.38 \\
0.4 & 0.2 & 0.4 \\
0 & 0.2 & 0.8
\end{array}\right)
$$

Starting from the same individual utilities reported in Table 3, by relying on the obtained SIN, it is possible to estimate missing FPRs elements (corresponding to the unknown utilities) with the iterative application of equations (15)-(18). The obtained FPRs are reported in Table 4 (second row) and should be compared to the estimated values assuming indifference of preference between unrated items and other items (first row of the same table). The obtained FPRs are then evolved according to the process described in section 4.2 to simulate the effects of social interaction. After 6 iterations, all individual FPRs converge to the following one:

$$
P=\left(\begin{array}{lllllll}
0.50 & 0.50 & 0.26 & 0.30 & 0.34 & 0.28 & 0.29 \\
0.50 & 0.50 & 0.27 & 0.30 & 0.30 & 0.28 & 0.29 \\
0.74 & 0.73 & 0.50 & 0.53 & 0.52 & 0.51 & 0.53 \\
0.70 & 0.70 & 0.47 & 0.50 & 0.51 & 0.48 & 0.50 \\
0.66 & 0.70 & 0.48 & 0.49 & 0.50 & 0.47 & 0.56 \\
0.72 & 0.72 & 0.49 & 0.52 & 0.53 & 0.50 & 0.52 \\
0.71 & 0.71 & 0.47 & 0.50 & 0.44 & 0.48 & 0.50
\end{array}\right) .
$$

Then, it is possible to obtain the group preference of items through equation (8) and, with equation (10), their group utility as reported in Table 5 (second row). Thus, according to the influence-based model, the top-ranked items for the group are: $x_{39}$ and $x_{77}$ followed by $x_{67}$ and $x_{46}$. Compared with the results obtained by the Standard GDM-based model, we notice that $x_{39}$ is now preferred over $x_{77}$ even in presence of a higher average of individual utilities. This is because $x_{39}$ is preferred by users $u_{123}$ and $u_{467}$ whom, according to the SIN, are more influencing than $u_{335}$ (who prefers $x_{77}$ instead). It should also be noted the good position of $x_{67}$ due to the fact that the opinion of the influencing member $u_{467}$ (initially unknown) is formed on that of $u_{335}$ (that likes $x_{67}$ ) disregarding that of the untrusted member $u_{123}$ (that dislikes $x_{67}$ ). 


\begin{tabular}{c|ccccccc}
\hline \hline \multirow{2}{*}{ Model } & \multicolumn{7}{|c}{ Estimated group utilities } \\
\cline { 2 - 8 } & $x_{12}$ & $x_{25}$ & $x_{39}$ & $x_{46}$ & $x_{67}$ & $x_{77}$ & $x_{89}$ \\
\hline Standard GDM-based & 0.51 & 0.10 & 0.91 & 0.76 & 0.31 & 1.00 & 0.37 \\
Influence-based & 0.11 & 0.10 & 1.00 & 0.89 & 0.89 & 0.97 & 0.85 \\
\hline \hline
\end{tabular}

Table 5. Comparison of group utilities estimated with the GDM-based models

Figure 3 shows the evolution of the individual preference degree associated to each item for group users, which elucidates the convergence process versus the final group preferences (before normalization). The $x$-axis represents the number of performed iterations while the $y$-axis shows the dominance degree of each alternative for each expert at a given iteration (obtained by applying equation (8) on individual FPRs). Different colors correspond to different alternatives, as shown on the right vertical line. This figure allows to easily perceive the final ranking as well as the process dynamics that led to the generation of the final decision. For example, it can be noticed that the most controversial alternatives are $x_{12}$ and $x_{49}$ since the convergence on them is reached later than for the other alternatives.

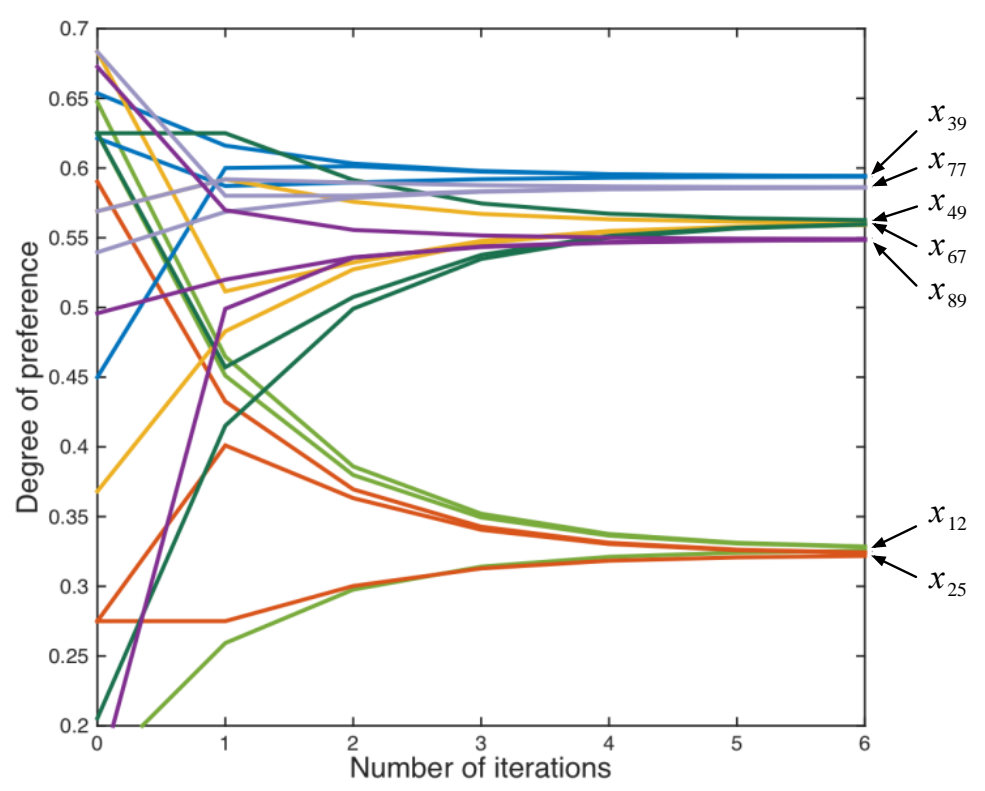

Figure 3. Evolution of group item utilities based on the influence model

\section{Final Remarks}

In this paper, the problem of group recommendation has been addressed through the definition of an original approach based on the adaptation and extension of fuzzy models for GDM. While in GDM, a group of decision makers evaluate a set of alternatives with the aim of selecting the best one to adopt, in GRS the system selects, from a given catalogue, the set of items that best fit the preferences of all (or the majority of) users belonging to a group. While the majority of existing GRSs just use individual users' preferences to estimate that of the group, the proposed approach, by using influence-based GDM models, also considers the interpersonal trust and personality of group members.

Information on interpersonal trust can be obtained by analyzing implicit data contained in social networks according to the models developed in $[54,7]$. In addition, personality traits can be predicted by analyzing the language used in social media according to the methods proposed in [59, 60,64]. Starting from this information, a SIN is generated according to $[9,51,52]$ and, subsequently, used to 
complete and evolve users' preferences toward a shared solution to be adopted at group level. The results of an in-silico simulation of the proposed models have been also discussed and the differences between standard GDM-based and social-based models for group recommendation have been pointed out.

The simulation of the natural evolution of group members' opinions stemming from discussion is a distinctive feature of the proposed model. In addition to other existing trust-based RS, it combines both interpersonal trust and personality in the concept of social influence. We believe that, taking both factors into account, it is possible to build a more accurate representation of the item selection process in practice, leading to better recommendations. A thorough experimentation of the proposed model with real users is planned as future work to measure the level of acceptance of the generated recommendations.

\section{References}

[1] G. Adomavicius and A. Tuzhilin, "Toward the next generation of recommender systems: a survey of the state-ofthe-art and possible extensions," IEEE Transactions on Knowledge and Data Engineering, vol. 17, no. 6, pp. 734749, 2005.

[2] N. Capuano, M. Gaeta, P. Ritrovato and S. Salerno, "Elicitation of Latent Learning Needs Through Learning Goals Recommendation," Computers in Human Behavior, vol. 30, pp. 663-673, 2014.

[3] N. Capuano, G. R. Mangione, A. Pierri and S. Salerno, "Learning Goals Recommendation for Self Regulated Learning," International Journal of Engineering Education, vol. 28, no. 6, pp. 1373-1379, 2012.

[4] A. Tejeda-Lorente, C. Porcel, E. Peis, R. Sanz and E. Herrera-Viedma, "A quality based recommender system to disseminate information in a University Digital Library," Information Sciences, vol. 261, pp. 52-69, 2014.

[5] T. De Pessemier, S. Dooms and L. Martens, "Comparison of group recommendation algorithms," Multimedia Tools and Applications, vol. 72, no. 3, pp. 2497-2541, 2014.

[6] J. Masthoff, "Group Recommender Systems: Combining Individual Models," in Recommender Systems Handbook, Springer, 2011, pp. 677-702.

[7] L. Quijano-Sanchez, J. A. Recio-Garcia, B. Diaz-Agudo and G. Jimenez-Diaz, "Social factors in group recommender systems," ACM Transactions on Intelligent Systems and Technology, vol. 4, no. 1, pp. 8:1-8:30, 2013.

[8] F. J. Cabrerizo, F. Chiclana, R. Al-Hmouz, A. Morfeq, A. S. Balamash and E. Herrera-Viedma, "Fuzzy decision making and consensus," Journal of Intelligent and Fuzzy Systems, vol. 29, no. 3, pp. 1109-1118, 2015.

[9] N. Capuano, F. Chiclana, H. Fujita, E. Herrera-Viedma and V. Loia, "Fuzzy Group Decision Making with Incomplete Information Guided by Social Influence," IEEE Transaction of Fuzzy Systems, 2017. doi: 10.1109/TFUZZ.2017.2744605.

[10] J. Wu, F. Chiclana, H. Fujita and E. Herrera-Viedma, "A visual interaction consensus model for social network group decision making with trust propagation," Knowledge Based-Systems, vol. 122, pp. 39-50, 2017.

[11] L. Perez, F. Mata, F. Chiclana, G. Kou and H.-V. E, "Modelling influence in group decision making," Soft Computing, vol. 20, no. 4, pp. 1653-1665, 2016.

[12] J. McCarthy and T. Anagnost, "MusicFX: an arbiter of group preferences for computer supported collaborative workouts," in Proceedings of the ACM conference on Computer Supported Cooperative Work, New York, USA, 1998.

[13] A. Crossen, J. Budzik and K. J. Hammond, "Flytrap: intelligent group music recommendation," in Proceedings of the 7th international conference on Intelligent User Interfaces, New York, USA, 2002.

[14] M. O'Connor, D. Cosley, J. A. Konstan and J. Riedl, "Polylens: a recommender system for groups of users," in Proceedings of the 7th conference on European conference on computer supported cooperative work, Norwell, USA, 2001.

[15] Z. Yu, X. Zhou, Y. Hao and J. Gu, "TV Program Recommendation for Multiple Viewers Based on user Profile Merging," User Modeling and User-Adaped Interaction, vol. 16, no. 1, pp. 63-82, 2006. 
[16] D. Goren-Bar and O. Glinansky, "Family stereotyping-a model to filter tv programs for multiple viewers," in Proceedings of the 2nd workshop on personalization in future tv, Malaga, Spain, 2002.

[17] S. Berry, S. Fazzio, Y. Zhou, B. Scott and L. Francisco-Revilla, "Netflix Recommendations for Groups," in Proceedings of the 73rd Annual Meeting of the American Society for Information Science and Technology, Pittsburgh, USA, 2010.

[18] A. Jameson, S. Baldes and T. Kleinbauer, "Two methods for enhancing mutual awareness in a group recommender system," in Proceedings of the working conference on Advanced Visual Interfaces, New York, USA, 2004.

[19] K. McCarthy, M. Salamo, L. Coyle, L. McGinty, B. Smyth and P. Nixon, "Cats: a synchronous approach to collaborative group recommendation," in Florida Artificial Intelligence Research Society Conference, 2006.

[20] J. McCarthy, "Pocket restaurant finder: a situated recommender system for groups," in Proceedings of the ACM Conference on Human Factors in Computing Systems, Minneaoplis, USA, 2002.

[21] L. Ardissono, A. Goy, G. Petrone, M. Segnan and P. Torasso, "Tailoring the recommendation of tourist information to heterogeneous user groups," Lecture Notes in Computer Science, vol. 2266, pp. 228-231, 2002.

[22] R. R. Sinha and K. Swearingen, "Comparing recommendations made by online systems and friends," in Proceedings of the DELOS Workshop: Personalisation and Recommender Systems in Digital Libraries, 2001.

[23] J. Golbeck, "Generating predictive movie recommendations from trust in social networks," in Proceedings of the 4th International Conference on Trust Management, 2006.

[24] N. Verbiest, C. Cornelis, P. Victor and E. Herrera-Viedma, "Trust and distrust aggregation enhanced with path length incorporation," Fuzzy Sets and Systems, vol. 202, no. 1, pp. 61-74, 2012.

[25] J. Golbeck, "Combining provenance with trust in social networks for semantic web content filtering," Lecture Notes in Computer Science, vol. 4145, pp. 101-108, 2006.

[26] F. Chiclana, F. Herrera and E. Herrera-Viedma, "Integrating three representation models in fuzzy multipurpose decision making based on fuzzy preference relations," Fuzzy Sets and Systems, vol. 97, no. 1, pp. 33-48, 1998.

[27] N. Capuano, V. Loia and F. Orciuoli, "A Fuzzy Group Decision Making Model for Ordinal Peer Assessment," IEEE Transactions on Learning Technology, vol. 10, no. 2, pp. 247-259, 2017.

[28] N. Capuano and F. Orciuoli, "Application of Fuzzy Ordinal Peer Assessment in Formative Evaluation," in Advances on P2P, Parallel, Grid, Cloud and Internet Computing, vol. 13, Springer, 2017, pp. 555-564.

[29] J. Castro, F. J. Quesada, I. Palomares and L. Martinez, "A Consensus-Driven Group RecommenderSystem," International Journal of Intelligent Systems, vol. 30, pp. 887-906, 2015.

[30] J. Castro, O. Cordon and L. Martinez, "CLG-REJA: A Consensus Location-aware group recommender system for Restaurants," in 9th ACM Conference on Recommender Systems, Vienna, Austria, 2015.

[31] C. C. Aggarwal, Recommender Systems: The Textbook, Springer, 2016.

[32] J. A. Konstan, B. N. Miller, D. Maltz, J. L. Herlocker, L. R. Gordon and J. Riedl, "Grouplens: applying collaborative filtering to usenet news," Communications of ACM, vol. 40, no. 3, pp. 77-87, 1997.

[33] L. Ungar and D. Foster, "A Formal Statistical Approach to Collaborative Filtering," in Proceedings of the Conference on Automated Learning and Discovery, Pittsburgh, USA, 1998.

[34] J. L. Herlocker, J. A. Konstan and J. Riedl, "Explaining collaborative filtering recommendations," in Proceedings of the 2000 ACM conference on computer supported cooperative work, New York, USA, 2000.

[35] J. Masthoff, "Group modeling: selecting a sequence of television items to suit a group of viewers," User Modeling and User-Adapted Interaction, vol. 14, no. 1, pp. 37-85, 2004.

[36] F. Cabrerizo, M. Ureña, W. Pedrycz and E. Herrera-Viedma, "Building consensus in group decision making with an allocation of information granularity," Fuzzy Sets and Systems, vol. 255, pp. 115-127, 2014.

[37] W. Liu, Y. Dong, F. Chiclana, F. Cabrerizo and E. Herrera-Viedma, "Group decision-making based on heterogeneous preference relations with self-confidence," Fuzzy Optimization and Decision Making, vol. 16, no. 4, pp. 429-447, 2017.

[38] G. Li, G. Kou and Y. Peng, "A Group Decision Making Model for Integrating Heterogeneous Information," IEEE Transactions on Systems, Man, and Cybernetics: Systems, 2018, doi: 10.1109/TSMC.2016.2627050.

[39] W. Wu and G. Kou, "A group consensus model for evaluating real estate investment alternatives," Financial Innovation, vol. 2, no. 8, 2016. 
[40] J. Lu, G. Zhang, D. Ruan and F. Wu, Multi-Objective Group Decision Making, Methods, Software and Applications with Fuzzy Set Techniques, World Scientific, 2007.

[41] W. Pedrycz, P. Ekel and R. Parreiras, Fuzzy Multicriteria Decision-Making: Models, Methods and Applications, John Wiley \& Sons, 2010.

[42] J. Wu, F. Chiclana, H. Fujita and E. Herrera-Viedma, "A minimum adjustment cost feedback mechanism based consensus model for group decision making under social network with distributed linguistic trust," Information Fusion, vol. 41, pp. 232-242, 2018.

[43] W. Liu and H. Liao, "A Bibliometric Analysis of Fuzzy Decision Research During 1970-2015," International Journal of Fuzzy Systems, vol. 19, no. 1, pp. 1-14, 2017.

[44] Y. M. Wang and Z. P. Fan, "Fuzzy preference relations: Aggregation and weight determination," Computers \& Industrial Engineering, vol. 53, no. 1, pp. 163-172, 2007.

[45] R. R. Yager, "Families of OWA operators," Fuzzy Sets and Systems, vol. 59, no. 2, pp. 125-148, 1993.

[46] L. A. Zadeh, "A Computational Approach to Fuzzy Quantifiers in Natural Languages," Computers and Mathematics with Applications, vol. 9, pp. 149-184, 1983.

[47] H. Liao, Z. Xu, E. Herrera-Viedma and F. Herrera, "Hesitant Fuzzy Linguistic Term Set and Its Application in Decision Making: A State-of-the-Art Survey," International Journal of Fuzzy Systems, 2017, doi: 10.1007/s40815017-0432-9.

[48] X. Wu and H. Liao, "An approach to quality function deployment based on probabilistic linguistic term sets and ORESTE method for multi-expert multi-criteria decision making," Information Fusion, vol. 43, pp. 13-26, 2018.

[49] F. Chiclana, F. Herrera, E. Herrera-Viedma and M. C. Poyatos, "A classification method of alternatives for multiple preference ordering criteria based on fuzzy majority," Journal of Fuzzy Mathematics, vol. 4, pp. 801-813, 1996.

[50] N. Capuano, F. Chiclana, H. Fujita, E. Herrera-Viedma and V. Loia, "Fuzzy Rankings for Preferences Modeling in Group Decision Making," International Journal of Intelligent Systems, 2018, doi: 10.1002/int.21997.

[51] M. H. De Groot, "Reaching a consensus," Journal of American Statistical Association, vol. 69, pp. 118-121, 1974.

[52] N. Friedkin and E. Johnsen, "Social Influence Networks and Opinion Change," Advances in Group Processes, vol. 16, no. 1, pp. 1-29, 1999.

[53] D. Z. Levin, R. Corss and L. C. Abrams, "The strength of weak ties you can trust: the mediating role of trust in effective knowledge transfer," Management Science, vol. 50, pp. 1477-1490, 2004.

[54] E. Gilbert and K. Karahalios, "Predicting tie strength with social media," in Proceedings of the 27th International Conference on Human Factors in Computing Systems, Boston, USA, 2009.

[55] K. Thomas and R. Kilmann, Thomas-Kilmann Conflict Mode Instrument, New York, USA: Tuxedo, 1974.

[56] J. E. Barbuto, K. A. Phipps and Y. Xu, "Testing relationships between personality, conflict styles and effectiveness," International Journal of Conflict Management, vol. 21, no. 4, pp. 434-447, 2010.

[57] L. C. Messarra, S. Karkoulian and A. N. El-Kassar, "Conflict resolution styles and personality: The moderating effect of generation $\mathrm{X}$ and $\mathrm{Y}$ in a non-Western context," International Journal of Productivity and Performance Management, vol. 65, no. 6, pp. 792-810, 2016.

[58] F. De Fruyt, R. R. McCrae, Z. Szirmák and J. Nagy, "The Five-Factor personality inventory as a measure of the FiveFactor Model: Belgian, American, and Hungarian comparisons with the NEO-PI-R," Assessment, vol. 11, no. 3, pp. 207-215, 2004.

[59] H. A. Schwartz, J. C. Eichstaedt, M. L. Kern, L. Dziurzynski, S. M. Ramones, M. Agrawal, A. Shah, M. Kosinski, D. Stillwell, S. M. E. P and U. L. H, "Personality, Gender, and Age in the Language of Social Media: The OpenVocabulary Approach," PLOS ONE, vol. 8, no. 9, 2013.

[60] M. Kosinski, D. Stillwell and T. Graepel, "Private traits and attributes are predictable from digital records of human behavior," Proceedings of the National Academy of Sciences of the United States of America, vol. 110, no. 15, pp. 5802-5805, 2013.

[61] J. L. Herlocker, J. A. Konstan, T. L. G and J. T. Riedl, "Evaluating collaborative filtering recommender systems," ACM Transactions on Information Systems, vol. 22, no. 1, pp. 5-53, 2004.

[62] F. Chiclana, E. Herrera-Viedma, F. Herrera and S. Alonso, "Some induced ordered weighted averaging operators and their use for solving group decision-making problems based on fuzzy preference relations," European Journal of Operational Research, vol. 182, no. 1, pp. 383-399, 2007. 
[63] S. Alonso, E. Herrera-Viedma, F. Chiclana and F. Herrera, "Individual and Social Strategies to Deal with Ignorance Situations in Multi-Person Decision Making," International Journal of Information Technology and Decision Making, vol. 8, no. 2, pp. 313-333, 2009.

[64] N. Capuano, G. D’Aniello, A. Gaeta and S. Miranda, "A personality based adaptive approach for information systems," Computers in Human Behavior, vol. 44, pp. 156-165, 2015. 